\title{
Status of Air Shower Simulations
}

\author{
S. Ostapchenko \\ NTNU, Institut for fysikk, 7021 Trondheim, Norway \\ D.V. Skobeltsyn Institute of Nuclear Physics, Moscow State University, 119992 Moscow, \\ Russia
}

\begin{abstract}
The present status of extensive air shower (EAS) simulation procedures is reviewed. The advantages of combining numerical and Monte Carlo methods for the description of EAS development are discussed. Physics content of cosmic ray interaction models is briefly described and their predictions are compared to the first LHC data. Finally, some outstanding puzzles related to cosmic ray composition at the "ankle" energies are analyzed.
\end{abstract}

\section{Introduction}

Over the last two decades, simulations of extensive air shower (EAS) development have become an important ingredient of experimental analysis of high energy cosmic ray (CR) data. The complexity of the corresponding procedures is related to the fact that measured EAS characteristics have a very indirect relation to the properties of the primary CR particles, resulting from a multi-step nuclear-electro-magnetic cascade development. Air shower simulations can thus be improved in two directions: i) towards higher precision and/or efficiency of the calculations, ii) regarding the physics content.

Concerning the former, applying EAS simulation procedures to showers induced by ultra-high energy cosmic rays (UHECR), one faces the problem of enormous calculation time required. Thus, one has to care about an optimization of the shower modeling, in order to obtain sufficiently high simulation statistics, and about keeping a high accuracy for calculations of both average EAS characteristics and their distributions.

Coming to the latter, of special importance is a correct description of the cascade of nuclear interactions of the hadronic component of air showers, which acts as a backbone of EAS. On the other hand, the corresponding 
theoretical models remain just phenomenological ones and involve relatively large number of adjustable parameters. Thus, further model development related both to improvements of the corresponding theoretical description and to retuning of model parameters with new accelerator data is desirable.

\section{EAS simulation procedures}

The most transparent approach to EAS simulation is the direct modeling of the shower development, as realized e.g. in the CORSIKA program [1]: The propagation in the atmosphere and interactions of each particle are traced using Monte Carlo (MC) methods. The approach has a natural restriction: With the number of cascade particles rising proportionally to the energy of the primary cosmic ray, so does the computing time required. Hence, extension of the procedure to very high energies requires a certain optimization of the method, e.g. employing weighted-sampling: Only a number of representative particles among all the secondaries produced per interaction is traced further by a code; each of those particles acquires thus some weight.

A classical example is the Hillas's "thinning" method [2], where a single particle per interaction is chosen with the probability $E_{s} / E_{p}, E_{s}$ being the energy of the given secondary and $E_{p}$ - the one of the primary particle. Correspondingly, the weight of the chosen secondary is $w_{s}=E_{p} / E_{s} w_{p}, w_{p}$ being the weight of the primary. Though the method works well for average EAS characteristics, it introduces artificial fluctuations in the distributions of air shower observables, as discussed e.g. in [3]. The solution to the problem was to impose a restriction on maximal weights [3], in order to reduce the magnitude of artificial fluctuations, and to complement the method by an "unthinning" procedure [4]. The latter allows one to convert the distribution of "weighted" particles coming from the "thinned" EAS simulation to a realistic particle distribution and to reduce artificial fluctuations to a tolerable level. However, the simulation procedure remains time-consuming - as one has to find a balance between a sparser "thinning" and not too large weights.

On the other hand, the efficiency and the accuracy of air shower modeling can be significantly improved combining $\mathrm{MC}$ and numerical methods, as is done in the CONEX [5] and SENECA [6] codes. Indeed, as EAS fluctuations are dominated by interactions and the propagation in the atmosphere of both the primary $\mathrm{CR}$ particle and of first few generations of the most energetic secondaries, one can apply a two-step procedure: Explicit MC simulation of the initial stage of the shower and numerical description of secondary 
hadronic and electro-magnetic $(\mathrm{e} / \mathrm{m})$ cascades, based on the solutions of the corresponding cascade equations 1 For a number of applications, like calculations of fluorescence and Cherenkov radiation profiles of air showers, the so-obtained one-dimensional EAS modeling is sufficient. In the more general case, when one is interested in the signal in ground-based detectors, a three-step procedure is applied [6], which includes MC modeling of both the highest and the lowest energy part of EAS while intermediate energy range is described numerically. Treating most of the cascade with numerical methods, one improves the efficiency of the procedure and enhances its accuracy.

\section{Hadronic interaction models}

As discussed in the Introduction, the least certain part of EAS simulation procedures is the treatment of hadronic cascades in the atmosphere, which involves phenomenological models of hadronic and nuclear interactions.

Contemporary CR interaction models, like EPOS [7], QGSJET [8] and QGSJET-II [9], and SIBYLL [10], are characterized by a similar physics content, being designed to treat general hadronic collisions, which involves both nonperturbative "soft" and "hard" parton processes. Soft physics is usually described within the Reggeon Field Theory framework as soft Pomeron exchanges; hard parton dynamics is treated within the DGLAP formalism and implemented in the models either following the so-called minijet approach [11] or the qualitatively similar "semihard Pomeron" scheme [12]. Despite these general similarities, the models diverge in their predictions, which is both due to technical differences in the implementation of the above-discussed approaches and, especially, due to different treatments of non-linear interaction effects related to parton shadowing and saturation.

Around the energy of the CR knee, EAS characteristics obtained using different models are relatively close to each other. This is both due to the model calibration to similar sets of accelerator data and due to ongoing model tests by air shower experiments, notably by KASCADE [13], which resulted in serious improvements of certain models. In the discussed energy range, the observed EAS characteristics are rather well reproduced by simulations under reasonable assumptions on the CR composition, although certain contradictions persist and composition studies bear model-dependence [14].

\footnotetext{
${ }^{1}$ In SENECA, the numerical solution is employed for hadronic cascades while a pretabulation is used for $\mathrm{e} / \mathrm{m}$ cascades.
} 
The situation changes drastically at higher energies $\left(E_{0}>10^{18} \mathrm{eV}\right)$, where model predictions diverge noticeably and certain important observations by air shower experiments can not be explained by the present models.

\section{First measurements at the Large Hadron Collider}

The first LHC data have a strong impact on EAS simulation procedures and on the interpretation of CR observations. Apart from providing additional constraints for hadronic interaction models, measurements of secondary particle production by the CMS and ALICE collaborations gave no evidence on a more rapid energy rise of the multiplicity of hadronic collisions than predicted by the present $\mathrm{CR}$ interaction models. This is illustrated in Fig. 1, where the CMS data on the pseudorapidity density of charged particles in $p p$ collisions are compared to the corresponding results of $\mathrm{MC}$ models, the latter being obtained applying the experimental trigger to the $\mathrm{MC}$ generated hadronic final states. Clearly, the data are bracketed by the model predictions.

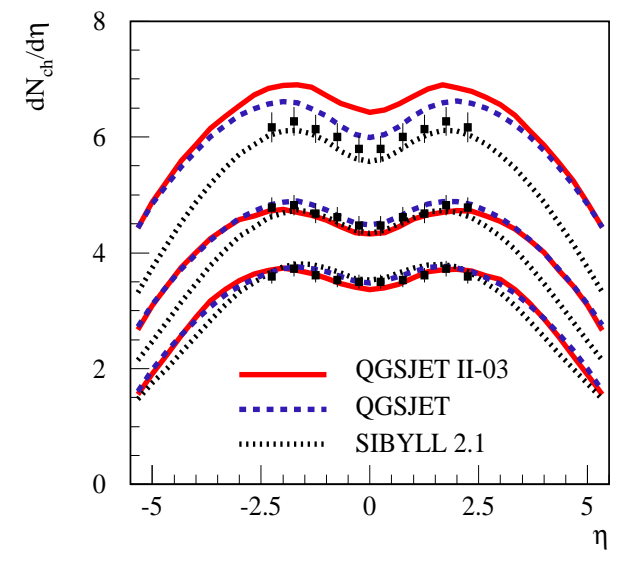

Figure 1: Predictions of CR interaction models for $d N_{\mathrm{ch}} / d \eta$ for different c.m. energies (from up to bottom: $\sqrt{s}=7,2.38$, $0.9 \mathrm{TeV})$ compared to CMS data [15].

\section{UHECR puzzles}

Historically, the first strong indication on a much higher EAS muon content than predicted by simulations has been observed in the HiRes-Mia analysis [16] for $\mathrm{CR}$ energies $E_{0}>10^{17} \mathrm{eV}$. More recently, the Pierre Auger collaboration has demonstrated using 4 different methods that experimental data favor a much higher (by a factor $\sim 1.5$ ) number of muons $N_{\mu}$ at ground than predicted e.g. by the QGSJET-II model [17]. Such a strong $N_{\mu}$ enhancement can not be achieved with the present CR interaction models, as it would require to increase the multiplicity of proton-air and pion-air collisions by an order of magnitude over a wide range of energies [18, 19]. On the other hand, the data are marginally consistent with simulation results for iron-induced air showers [17]. As the measurements refer mainly to 
primary energies below $10 \mathrm{EeV}$, the required change to an iron-dominated composition has to occur around the ankle of the CR spectrum.

Another striking result obtained was the sharp decrease of the width of the shower maximum distribution $\operatorname{RMS}\left(X_{\max }\right)$ at $E_{0}>10^{18} \mathrm{eV}[20$ ]. As discussed in 21], $\operatorname{RMS}\left(X_{\max }\right)$ is an almost model-independent measure of the CR composition. Indeed, for proton-induced EAS this quantity is mainly defined by the mean free pass of the proton $\lambda_{p} \sim 1 / \sigma_{p-\text { air }}^{\text {inel }}$, which sets the lower limit on the $\operatorname{RMS}\left(X_{\max }^{p}\right)$ around $50 \mathrm{~g} / \mathrm{cm}^{2}$. Fluctuations related to the geometry of $p$-air interactions (higher/smaller inelasticity for "central"/peripheral collisions) can only increase the corresponding value. On the other hand, in case of Fe-induced EAS, $\operatorname{RMS}\left(X_{\max }^{\mathrm{Fe}}\right)$ is rather dominated by the fluctuations of the collision geometry, primarily, via the variations of the number of "wounded" projectile nucleons (which participate in particle production) and via the fragmentation of the nuclear spectator part [22]. Even for extreme assumptions, $\operatorname{RMS}\left(X_{\max }^{\mathrm{Fe}}\right)$ can not exceed some $30 \mathrm{~g} / \mathrm{cm}^{2}$.

The observed decrease of $\operatorname{RMS}\left(X_{\max }\right)$ from $\sim 55 \mathrm{~g} / \mathrm{cm}^{2}$ at $1 \mathrm{EeV}$ to $\sim 30$ $\mathrm{g} / \mathrm{cm}^{2}$ at $30 \mathrm{EeV}$ may be naively interpreted as a change from a $p$-dominated to an Fe-dominated composition in the discussed energy range [20]. In reality, as correctly noticed in [23, 24], adding a small admixture of iron nuclei to the pure proton composition increases the width of the distribution. As an illustration, in Fig. 2 we compare the Pierre Auger data on $\operatorname{RMS}\left(X_{\max }\right)$ with EAS simulation results (using QGSJET-II) considering a simple 2component $(p+F e)$ composition and assuming the partial abundances $f_{i}$ to change smoothly between 1 and $30 \mathrm{EeV}$ : $f_{p}(E)=f_{p}(1 \mathrm{EeV})[1-\lg (E / \mathrm{EeV}) / 1.5]$, $f_{\mathrm{Fe}}(E)=1-f_{p}(E)$. Comparing the cases $f_{p}(1 \mathrm{EeV})=1$ and $f_{p}(1 \mathrm{EeV})=$ 0.4 , we see that it is the latter choice which is supported by the data, i.e. iron dominance at the ankle is favored.

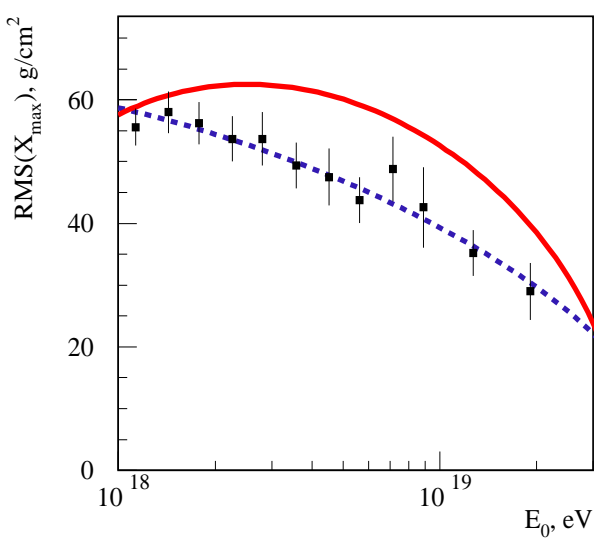

Figure 2: $\operatorname{RMS}\left(X_{\max }\right)$ for an energydependent CR composition (as described in the text): $f_{p}(1 \mathrm{EeV})=1$ (solid) and $f_{p}(1 \mathrm{EeV})=0.4$ (dashed); points Pierre Auger data [20].

On the other hand, a heavy CR composition in the EeV energy range is at variance with HiRes and Pierre Auger data on the average $X_{\max }$, which are consistent with the proton-dominance. In order to reconcile the latter 
with a heavy composition, a much deeper (than presently predicted) shower penetration in the atmosphere has to be assumed. In practical terms this would require a factor of 2 decrease for $\sigma_{p-\text { air }}^{\text {inel }}$ compared to the current model predictions [19], which would correspond to a similar reduction for the total $p p$ cross section at $\sqrt{s} \sim 6 \mathrm{TeV}$, i.e. only slightly above the Tevatron energy. Such a sudden fall down of $\sigma_{p p}^{\text {tot }}$ would imply very exotic physics.

\section{Conclusions}

Significant progress in the modeling of air showers has been achieved in recent years. Combing numerical and $\mathrm{MC}$ methods, as realized in the CONEX and SENECA codes, allowed one to increase the efficiency and the accuracy of EAS simulations. The description of hadronic collisions by the corresponding MC models has been considerably improved, particularly, concerning the treatment of non-linear interaction effects. Predictions of various hadronic MC models have converged to each other, both due to the improved theoretical description and due to model tests by EAS experiments.

The first LHC data on secondary hadron production in $p p$ collisions provide no indication on a more rapid energy rise of the multiplicity than predicted by the present CR interaction models. Thus, a strong rise of EAS muon content at $1 \div 100 \mathrm{PeV}$ is not supported by the collider observations.

The strong enhancement of EAS muon content and the sharp decrease of the width of $X_{\max }$ distributions, as observed by the Pierre Auger collaboration above $1 \mathrm{EeV}$, can not be explained in the framework of the present $\mathrm{CR}$ interaction models, unless an iron dominance of the CR composition at the ankle is assumed. The latter assumption is however in a strong conflict with $X_{\max }$ measurements at $1 \div 10 \mathrm{EeV}$ by all the experiments working in this energy range. In turn, the data on average $X_{\max }$ can not be reconciled with a heavy $\mathrm{CR}$ composition without invoking very exotic physics.

Acknowledgements The author acknowledges discussions with Michael Kachelriess and a fellowship from the program Romforskning of Norsk Forsknigsradet.

\section{References}

[1] D. Heck et al., FZKA-60198, Forschungszentrum Karlsruhe (1998).

[2] M. Hillas, Nucl. Phys. Proc. Suppl. 52B (1997) 29.

[3] M. Kobal for the Pierre Auger Collab., Astopart. Phys. 15 (2001) 259. 
[4] P. Billoir, Astopart. Phys. 30 (2008) 270.

[5] T. Bergmann et al., Astopart. Phys. 26 (2007) 420.

[6] H.J. Drescher and G.R. Farrar, Phys. Rev. D 67 (2003) 116001.

[7] K. Werner, F.-M. Liu and T. Pierog, Phys. Rev. C 74, 044902 (2006).

[8] N.N. Kalmykov, S.S. Ostapchenko, and A.I. Pavlov, Bull. Russ. Acad. Sci. Phys. 58 (1994) 1966; Nucl. Phys. Proc. Suppl. 52B (1997) 17.

[9] S. Ostapchenko, Nucl. Phys. Proc. Suppl. 151 (2006) 143; Phys. Rev. D 74 (2006) 014026; AIP Conf. Proc. 928 (2007) 118.

[10] R.S. Fletcher et al., Phys. Rev. D 50 (1994) 5710; E.-J. Ahn et al., Phys. Rev. D 80 (2009) 094003.

[11] L. Durand and P. Hong, Phys. Rev. Lett. 58 (1987) 303.

[12] H.J. Drescher et al., J. Phys. G 25 (1999) L91; S. Ostapchenko et al., J. Phys. G 28 (2002) 2597.

[13] W.D. Apel et al. (KASCADE Collab.), J. Phys. G 34 (2007) 2581.

[14] T. Antoni et al. (KASCADE Collab.), Astopart. Phys. 24 (2005) 1.

[15] V. Khachatryan et al. (CMS Collab.), Phys. Rev. Lett. 105 (2010) 022002 .

[16] R.U. Abbasi et al. (HiRes Collab.), Astroph. J. 622 (2005) 910.

[17] A. Castellina for the Pierre Auger Collab., in: Proc. $31^{\text {st }}$ Int. Cosmic Ray Conf. (Lódź); arXiv:0906.2319.

[18] S. Ostapchenko, Czech. J. Phys. 56 (2006) A149.

[19] R. Ulrich et al., arXiv:0906.0418 [astro-ph.HE].

[20] J. Abraham et al. (Pierre Auger Collab.), Phys. Rev. Lett. 104 (2010) 091101.

[21] R. Aloisio et al., Phys. Rev. D 77 (2008) 025007.

[22] N.N. Kalmykov and S.S. Ostapchenko, Phys. Atom. Nucl. 56346 (1993). 
[23] D. Hooper, A. M. Taylor, Astopart. Phys. 33 (2010) 151.

[24] G. Wilk and Z. Wlodarczyk, arXiv:1006.1781 [astro-ph.HE]. 